Notiz über eine Classe symmetrischer Determinanten.

Von

M. Noethez in Erlangen.

Es liönnte von Interesse sein, die Classe symmetrischer Determinanten kennen zu lernen, welche die folgenden beiden Arten als specielle Fälle umfasst:

1) die bekannte*) Determinante $n^{\text {ten }}$ Grades, deren Reiben aus der ersten Reihe

$$
a_{0}, a_{1}, a_{2}, \cdots, a_{n-1}
$$

durch cyklische Vertauschung aller Elemente entstehen, also die Resultante der beiden Formen:

$$
\begin{aligned}
& f=\sum_{i=0}^{i=n-1} a_{i} x^{i}, \\
& \varphi=x^{n}-1 ;
\end{aligned}
$$

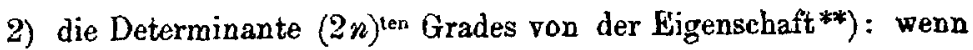
man die Elemente in vier Quadrate

$$
\begin{array}{ll}
A & B \\
C & D
\end{array}
$$

von je $2^{\mu-1}$ Reihen zusammenfasst, so stimmen die Elemente des Theils $A$ mit denen des gegenüberliegenden Theils $D$ genau überein, ebenso die von $B$ mit denen von $C$; dasselbe gilt für die Zerlegung dieser Theile in je 4 weitere Quadrate; ebenso für diese letzteren, etc.

Die diese beiden Arten umfassende allgemeinere Deterninante, welche bisher noch nicht bemerkt worden zu sein scheint, lässt sich ebenfalls als Resultante auffassen. Sei

$$
f=\sum_{i_{1}, i_{2}, \ldots, i_{\mu}} a_{i_{1} i_{2} \ldots i_{\mu}} x_{1}^{i_{1}} x_{2}^{i_{2}} \cdots x_{\mu}^{i_{\mu}}
$$

*) Vgl für die Litteratur Balt ze r'\& „Deterninanten“, 4. Auflage, \& 11., 1.,2., 3.

*) Vgl. Puchta, Denkschr. d. Wiener Akad., Bd. 38, p. 315, und meinen Aufsatz: Zur Theorie der Thetafunctionen etc., d. Annalen, XVL, p. 286; \$ 15. 
eine (nicht homogene) Form, welche in der Variabeln $x_{\rho}$ bis zum Grade

$$
n_{\rho}-1 \quad\left(n_{e}>1\right)
$$

$(\varrho=1,2, \cdots, \mu)$ aufsteigt; und weiter seien die Formen gegebev:

$$
\varphi_{1}=x_{1}^{n_{1}}-1, \quad \varphi_{2}=x_{2}^{n_{2}}-1, \cdots, \varphi_{\mu}=x_{\mu}^{n_{\mu}}-1 \text {. }
$$

Die Resultante dieser $\mu+1$ Formen wird:

wo

$$
R=\Pi_{\bullet} \cdot\left\{\sum_{i_{1}, i_{2}, \ldots, i_{\mu}} a_{i_{1} i_{2} \ldots i_{\mu}} \varepsilon_{1}^{i_{1}} \varepsilon_{2}^{i_{2}} \cdots \varepsilon_{\mu}^{i_{\mu}}\right\},
$$

und wo das Product $\Pi_{\text {e }}$ über alle

$$
\varepsilon_{\rho}^{n} \rho=1 \quad(\rho=1,2, \cdots, \mu),
$$

$$
n_{1} n_{2} \cdots n_{\mu}
$$

Werthsysteme der $\varepsilon$ auszudehneu ist. Formen

Die Resultante wird zugleich als Determinante $\Delta$ der $n_{1} n_{2} \ldots n_{\mu}$

$$
\varepsilon_{1}^{k_{1}} \varepsilon_{2}^{k_{2}} \cdots \varepsilon_{\mu^{\mu}}^{k_{\mu}} \sum a_{i_{1} i_{2} \ldots i_{\mu}} \varepsilon_{1}^{i_{1}} \varepsilon_{2}^{i_{2}} \cdots \varepsilon_{\mu^{\mu}}^{i_{\mu}},
$$

die als lineare in den $n_{1} n_{2} \cdots n_{\mu}$ Variabeln

$$
\varepsilon_{1}^{h_{1}} \varepsilon_{2}^{h_{2}} \cdots \varepsilon_{\mu}^{h_{\mu}}
$$

aufgefasst werden, erhalten. Dabei bilden die $\varepsilon_{1}, \varepsilon_{2}, \cdots, \varepsilon_{\mu}$ ein System einer $n_{1}^{\text {len }}, n_{2}^{\text {ten }}, \cdots, n_{\mu}^{\text {ten }}$ Wurzel der Einheit.

Wir denken uns dabei die $n_{1} n_{2} \cdots n_{\mu}$ Variabeln derart geordnet: zunächst kommen

$$
1, \varepsilon_{1}, \varepsilon_{1}^{2}, \cdots, \varepsilon_{1}{ }^{n_{1}-1} \text {; }
$$

alsdann diese $n_{1}$ bez. mit $\varepsilon_{2}, \varepsilon_{2}{ }^{2}, \cdots, \varepsilon_{2}{ }^{n_{2}-1}$ verbunden:

$$
\begin{gathered}
\varepsilon_{2}, \varepsilon_{1} \varepsilon_{2}, \varepsilon_{1}^{2} \varepsilon_{2}, \cdots, \varepsilon_{1}^{n_{1}-1} \varepsilon_{2}, \\
\cdot \cdot \cdot \cdot \cdot \cdot \cdot \cdot \cdot \cdot \cdot \cdot \cdot \cdot \cdot \cdot \cdot \cdot \varepsilon_{1}^{n_{1}-1} \varepsilon_{2}^{n_{2}-1} ;
\end{gathered}
$$

alsdann alle diese $n_{1} n_{2}$ der Reihe nach mit $\varepsilon_{3}$, dann mit $\varepsilon_{3}{ }^{2}, \ldots$, mit $\varepsilon_{3}{ }^{n_{3}-1}$ verbunden; etc. Ebenso denken wir uns die Factoren

$$
\varepsilon_{1}^{k_{1}} \varepsilon_{2}^{k_{2}} \cdots \varepsilon_{\mu}^{k_{\mu}}
$$

die vor den Zeichen $\Sigma$ in den obigen $n_{1} n_{2} \cdots n_{\mu}$ linearen Formen (5) stehen, geordnet. Die so geordnete Determinante $\Delta$ lässt sich folgendermassen definiren:

$\Delta$ ist eine symmetrische Determinante von $n_{1} n_{2} \cdots n_{\mu}$ Reihen. Theilt man $\Delta$ durch $n_{\mu}-1$ Horizontalstriche und ebensoviele Verticalstriche in $n_{\mu}^{2}$ Quadrate $\Delta^{\prime}$ von je $n_{1} n_{2} \cdots n_{\mu-1}$ Reihen, so gehen die $n_{\mu}$ Horizontalreihen dieser Quadrate aus der ersten durch Wiederholung derselben cyklischen Vertauschung hervor, indem man nämlich jedes der $n_{\mu}$ Quadrate der ersten Reihe um ein Glied vorrïckt. Theilt 
man weiter jedes dieser $n_{\mu}^{2}$ Quadrate $\Delta^{\prime}$ analog in je $n_{\mu-1}^{2}$ Quadrate $\Delta^{\prime \prime}$, so gehen auch die $n_{\mu-1}$ Reihen solcher Quadrate, welche in einem $\Delta^{\prime}$ enthalten sind, aus der ersten Reihe von $n_{\mu-1}$ Quadraten durch analoge cyklische Vertauschung hervor; ebenso bei der analogen Zertheilung jedes $\Delta^{\prime \prime}$ in je $n_{\mu-2}^{2}$ Quadrate, etc.

So hat man z. B. für $\mu=3$ und $n_{1}=2, n_{2}=2, n_{3}=3$ :

$\Delta=\left|\begin{array}{llllllllllll}a_{000} & a_{100} & a_{010} & a_{110} & a_{001} & a_{101} & a_{011} & a_{111} & a_{002} & a_{102} & a_{012} & a_{112} \\ a_{100} & a_{000} & a_{110} & a_{010} & a_{101} & a_{001} & a_{111} & a_{011} & a_{102} & a_{002} & a_{112} & a_{012} \\ a_{010} & a_{110} & a_{000} & a_{100} & a_{011} & a_{111} & a_{001} & a_{101} & a_{012} & a_{112} & a_{002} & a_{102} \\ a_{110} & a_{010} & a_{100} & a_{000} & a_{111} & a_{011} & a_{101} & a_{001} & a_{112} & a_{012} & a_{102} & a_{002} \\ a_{002} & a_{102} & a_{012} & a_{112} & a_{000} & a_{100} & a_{010} & a_{110} & a_{001} & a_{101} & a_{011} & a_{111} \\ a_{102} & a_{002} & a_{112} & a_{012} & a_{100} & a_{000} & a_{110} & a_{010} & a_{101} & a_{001} & a_{111} & a_{011} \\ a_{012} & a_{112} & a_{002} & a_{102} & a_{010} & a_{110} & a_{000} & a_{100} & a_{011} & a_{111} & a_{001} & a_{101} \\ a_{112} & a_{012} & a_{102} & a_{002} & a_{110} & a_{010} & a_{100} & a_{000} & a_{111} & a_{011} & a_{101} & a_{001} \\ a_{001} & a_{101} & a_{011} & a_{111} & a_{002} & a_{102} & a_{012} & a_{112} & a_{000} & a_{100} & a_{010} & a_{110} \\ a_{101} & a_{001} & a_{111} & a_{011} & a_{102} & a_{002} & a_{112} & a_{012} & a_{100} & a_{000} & a_{110} & a_{010} \\ a_{011} & a_{111} & a_{001} & a_{101} & a_{012} & a_{112} & a_{002} & a_{102} & a_{010} & a_{110} & a_{000} & a_{100} \\ a_{111} & a_{011} & a_{101} & a_{001} & a_{112} & a_{012} & a_{102} & a_{002} & a_{110} & a_{010} & a_{100} & a_{000}\end{array}\right|$

Für $\mu=1$ hat man den oben genannten Fall (1), für

den Fall (2).

$$
n_{1}=n_{2}=\cdots=n_{\mu}=2
$$

Analytisch lässt sich die Eigenschaft der allgemeinen Determinante $\Delta$ so aussprechen: Sei

\section{$c_{j j^{\prime}}$}

das Element von $\Delta$, welches in $\operatorname{der}(j+1)^{\text {ten }}$ Horizontal - und $\left(j^{\prime}+1\right)^{\text {ten }}$ Verticalreihe gelegen ist; man bringe $j$ und $j^{\prime}$ auf die Form:

$$
\left\{\begin{array}{l}
j=k_{1}+k_{2} n_{1}+k_{3} n_{1} n_{2}+\cdots+k_{\mu} n_{1} n_{2} \cdots n_{\mu-1}, \\
j^{\prime}=h_{1}+h_{2} n_{1}+h_{3} n_{1} n_{2}+\cdots+h_{\mu} n_{1} n_{2} \cdots n_{\mu-1},
\end{array}\right.
$$

wo

sind (oder

$$
\begin{aligned}
& 0 \leqq k_{\rho}<n_{\rho}, \\
& 0 \leqq h_{\rho}<n_{\rho}, \quad\left(\varrho=1,2, \cdots, n_{\mu}\right)
\end{aligned}
$$

$$
\begin{aligned}
& \frac{j-k_{1}-k_{2} n_{1}-k_{8} n_{1} n_{2}-\cdots-k_{Q-1} n_{1} n_{2} \cdots n_{Q-2}}{n_{1} n_{2} \cdots n_{Q-1}} \equiv k_{Q} \quad\left(\bmod n_{Q}\right), \\
& \left.\frac{j-h_{1}-h_{2} n_{1}-h_{8} n_{1} n_{2}-\cdots-h_{Q-1} n_{1} n_{2} \cdots n_{Q-2}}{n_{1} n_{2} \cdots n_{Q-1}} \equiv h_{Q} \quad\left(\bmod n_{Q}\right)\right) ;
\end{aligned}
$$


dann wird $c_{y}$, der Coefficient von $\varepsilon_{1}^{h_{1}} \varepsilon_{2}^{h_{2}} \cdots \varepsilon_{\mu}^{h_{\mu}}$ in dem Ausdruck:

d. h.

$$
\varepsilon_{1}^{k_{1}} \varepsilon_{2}^{k_{2}} \cdots \varepsilon_{\mu^{\mu}}^{k_{\mu}} \sum a_{i_{1} i_{2} \ldots i_{\mu}} \varepsilon_{1}^{i_{1}} \varepsilon_{2}^{i_{2}} \cdots \varepsilon_{\mu}^{i_{\mu}}
$$

$$
c_{j j}=a_{h_{1}-k_{1}, k_{2}-k_{2}, \ldots, h_{\mu}-k_{\mu}},
$$

wo $h_{Q}-k \bmod n_{Q}$ zu nehmen ist.

Die Determinante $\Delta$ hat bemerkenswerthe Eigenschaften. Als Resultante muss $\Delta$ mit $R(3)$ bis etwa auf einen numerischen Factor übereinstimmen. Dieser Factor wird $=1$, «Iso

$$
\Delta=R \text {, }
$$

wie man sieht, indem man alle Coefficienten von $f$, bis auf $a_{00} \ldots{ }_{0}$, zu 0 macht. Um direct nachzuweisen, dass $\Delta$ in das Product der $n_{1} n_{2} \cdots n_{\mu}$ linearen Factoren (3) zerfällt, multiplicire man die $\left(j^{\prime}+1\right)^{\text {te }}$ Verticalreihe mit $\varepsilon_{1}^{h_{1}} \varepsilon_{2}^{h_{2}} \cdots \varepsilon_{\mu^{\prime}}^{h_{u}}$, wo die $h_{Q}$ wie oben in (6) mit $j^{\prime} z u-$ sammenhängen, für jede $j^{\prime}$; addire alsdann alle Verticalreihen zur ersten und dividire endlich jede $(j+1)^{\text {te }}$ Horizontalreihe bez. durch $\varepsilon_{1}^{k_{1}} \varepsilon_{2}^{k_{2}} \cdots \varepsilon_{\mu}^{k_{\mu}}$, wo die $k_{\varrho}$ auch aus (6) zu nehmen sind. Die Elemente der ersten Verticalreihe werden alsdann alle einander gleich, und zwar

$$
=\sum a_{i_{1} i_{2} \ldots i_{\mu}} \varepsilon_{1}^{i_{1}} \varepsilon_{2}^{i_{2}} \cdots \varepsilon_{\mu}^{i_{\mu}}
$$

ein Ausdruck, der also als Factor in $\Delta$ enthalten ist. Da hierbei die $\varepsilon_{1}, \varepsilon_{2}, \cdots$ jedes Wurzelsystem (4) vorstellen können, so folgt die Gleichung (8).

Dic Unterdeterminanten nach zweien einander gleichen Elementen von $\Delta$ sind ebenfalls einander gleich, so dass dieselben eine der $\Delta$ analoge Determinante bilden.

Denn seien die Unterdeterminanten nach den Elementen der exsten Horizontalreihe bez. mit

bezeichnet; so bat man

$$
A_{i_{1} i_{2} \ldots i_{\mu}}
$$

$$
\left\{\begin{array}{l}
\sum a_{i_{1} i_{2} \ldots i_{\mu}} A_{i_{1_{1}} \ldots i_{\mu}}=\Delta, \\
\sum a_{i_{1} i_{2}{ }^{\prime} \ldots i_{\mu}} A_{i_{1} t_{2} \ldots i_{\mu}}=0
\end{array}\right.
$$

พ้อ

$$
i_{\rho}^{\prime} \equiv i_{\rho}-i_{\rho} \quad\left(\bmod . n_{\rho}\right), \quad(\varrho=1,2, \ldots, \mu),
$$

und die $k_{1}, k_{2}, \cdots, k_{\mu}$ irgend welche von den $i$ unabhängige Zahlen sind, die nicht alle $\equiv 0\left(\bmod n_{\rho}\right)$ sind. Nimmt man nun die Elemente von $\Delta$ so allgemein an, dass $\Delta$ nicht $=0$ ist, so bestimmen die $n_{1} n_{2} \cdots n_{\mu}$ Gleichungen (9) die $A_{i_{1} i_{2} \ldots i_{\mu}}$ eindeutig. Für die Unterdeterminanten nach den Elementen irgend einer andern Horizontalreihe bestehen aber genau dieselben Gleichungen (9), so dass also die- 
jenige, welche in der ersten der Gleichungen (9) mit $a_{i_{1} i_{2} \ldots i_{\mu}}$ multiplicirt ist, wiederum denselben Werth $A_{i_{1} i_{2}, \ldots i_{\mu}}$ erbält.

Für die Unterdeterminanten hat man die Relation:

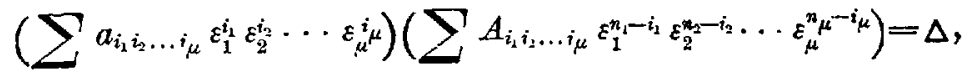

wo die $\varepsilon$ ein beliebiges Wurzelsystem von (4) vorstellen.

Denn um das Product zur Linken zu bilden, kann man zunächst jedes Glied

$$
a_{i_{1} i_{2} \ldots i_{\mu}} \varepsilon_{1}^{i_{1}} \varepsilon_{2}^{i_{2}} \cdots \varepsilon_{\mu}^{i_{\mu}}
$$

der ersten Summe mit dem entsprechenden Glied

$$
A_{i_{1} i_{2} \ldots i_{\mu}} \varepsilon_{1}^{n_{1}-i_{1}} \varepsilon_{2}^{n_{2}-i_{2}} \ldots \varepsilon_{\mu}^{n_{\mu}-i_{\mu}}
$$

der zweiten Summe multipliciren, was nach der ersten Gleichung (9) $\Delta$ liefert; sodann jedes Glied

$$
a_{i_{1}{ }^{\prime} i_{2}^{\prime} \ldots i_{\mu}^{\prime}} \varepsilon_{1}^{i_{1}^{\prime}} \varepsilon_{2}^{i_{2}^{\prime}} \cdots \varepsilon_{\mu}^{i_{\mu}^{\prime}}
$$

mit einem solchen

$$
A_{i_{1} i_{2} \ldots i_{\mu}} \varepsilon_{1}^{n_{1}-i_{1}} \varepsilon_{2}^{n_{2}-i_{2}} \ldots \varepsilon_{\mu}^{n_{\mu}-i_{\mu}},
$$

für welches die $i^{\prime}$ und $i$ wie oben in $(9)$, bei gegebenen $k_{\rho}$, zusammenhängen. Im letzteren Falle erhält man 0 nach (9), für jedes System $h_{e}$; womit die Gleichung (10) bewiesen ist.

Die Gleichung (10) liefert zugleich einen weiteren Beweis der Gleichung (8). Ausserdem giebt sie in Verbindung mit (8) eine Productdarstellung für die Summe:

$$
\sum A_{i_{1} i_{2} \ldots i_{\mu}} \varepsilon_{1}^{n_{1}-i_{1}} \varepsilon_{2}^{n_{2}-i_{2}} \ldots \varepsilon_{\mu}^{n_{\mu}-i_{\mu}}
$$

Mannheim, 31. December 1879. 